\title{
REDES DE PYMES: UNA VISIÓN DESDE LAS TEORÍAS DE CLUB Y DE EQUIPO
}

Maria Verónica Alderete*

$\mathrm{E}$ valor de las redes o alianzas entre empresas para el éxito empresarial es bastante reconocido. La cooperación es una estrategia para superar algunas debilidades de las empresas pequeñas y medianas (pymes) como el limitado acceso a los mercados externos y a la financiación o el rezago tecnológico, y así mejorar su competitividad.

En América Latina, Brasil es uno de los países que promueven las redes de pymes con apoyo de instituciones como el Servicio Brasilero de Apoyo a las Micro y Pequeñas Empresas (SEBRAE). La idea consiste en reunir empresas de segmentos afines en redes horizontales de cooperación que forman una entidad jurídica, sin cuotas de capital, en la que cada empresa mantiene su individualidad y su independencia legal. Esos programas crean un ambiente que estimula la capacidad empresarial y la asociación, con asesoraría técnica para formar, consolidar y desarrollar las redes.

En sentido amplio, el concepto de red entre firmas alude a grupos de empresas. La formación de redes, sean cuales sean la naturaleza y el tamaño de las empresas, la actividad económica, los objetivos y el formato final de la red, presupone que la empresa tiene límites. Como dice Penrose (1959) a este respecto: "toda empresa es un conjunto de recursos más o menos especializados, y no puede moverse con igual facilidad en todas las direcciones"; además, interactúa con otras empresas y agentes económicos. Las teorías que buscan expli-

\footnotetext{
* Doctora en Economía, investigador asistente del Instituto de Investigaciones Económicas y Sociales del Sur (IIESS-Conicet, Universidad Nacional del Sur), [mvealderete@gmail.com]. Fecha de recepción: 22 de febrero de 2013, fecha de modificación: 19 de julio de 2013, fecha de aceptación: 29 de abril de 2015. Sugerencia de citación: Alderete, M. V. "Redes de pymes: una visión desde las teorías de club y de equipo", Revista de Economía Institucional 17, 32, 2015, pp. 317-348. DOI: 10.18601/01245996.v17n32.11
} 
car la cooperación entre empresas varían según el enfoque teórico y metodológico: económico, gerencial, administrativo o sociológico. En particular, la teoría de clubes proporciona fundamentos teóricos para estudiar la asignación eficiente de bienes públicos impuros y determinar el tamaño óptimo de las alianzas.

Los clubes comparten ciertos recursos, bien sea un bien público impuro o algún atributo deseable de los miembros. En los consorcios de exportación, el recurso común puede ser la oficina de ventas y en las redes de compras, una bodega central. Pero el hecho de compartir puede provocar rivalidad o competencia por los beneficios a medida que aumenta el número de miembros, a expensas de la calidad del servicio recibido.

Por otro lado, un empeño colectivo de empresas pertenecientes a una red o alianza puede ser considerado como un caso de producción en equipo. La sinergia, la razón de ser de la existencia del equipo, puede llevar a que no se distinga la contribución individual al producto y a que sea imposible pagar a cada participante de acuerdo con su aporte. La teoría de equipos analiza el diseño de mecanismos para minimizar los problemas de información asimétrica.

En muchos casos que se observan en la realidad, las redes de empresas comparten características de equipos y de clubes. Por ejemplo, cuando las empresas participan en un equipo, la información privada de cada una se convierte en información común, y para el equipo es un bien club. Asimismo, cuando el desempeño de los clubes tiene costos de transacción surge el problema del azar moral, que está en el centro de la teoría de la producción en equipos.

Este artículo propone una taxonomía de casos observados de redes de pymes según se asemejen más a una actividad de trabajo en equipo o a la provisión de un bien club, es decir, un nuevo enfoque metodológico para analizar las redes de empresas desde el punto de vista de ambas teorías que complementa otras líneas teóricas. Esta propuesta no solo amplía la literatura sino que puede servir como herramienta para desarrollar las empresas y las redes. Primero hace una breve revisión de las teorías de clubes y de equipos. Luego sistematiza la información de algunas experiencias de redes de empresas de Argentina y Brasil según una u otra, o ambas. Después establece algunas dimensiones que se deben considerar cuando se interpreta una red de acuerdo con estas teorías. El análisis de los casos sugiere un nuevo marco teórico para analizar la cooperación y hacer breves recomendaciones de política. 


\section{METODOLOGÍA}

Para enmarcar el análisis de las redes primero se hace una revisión bibliográfica que sirve para establecer los criterios de referencia. Una vez establecidos los criterios o dimensiones que se derivan de las teorías de clubes y de equipos, se caracterizan algunos casos de redes de empresas conforme a estas dimensiones. La taxonomía se basa en redes de empresas de Argentina y Brasil seleccionadas por la autora de acuerdo con la disponibilidad de información ${ }^{1}$. En Brasil, los datos se obtuvieron durante la investigación doctoral en Campinas².

\section{MARCO TEÓRICO}

El concepto de red de empresas ha sido definido de varias maneras, según el enfoque teórico. Rauch y Casella (2001) hacen una buena revisión de las definiciones utilizadas por economistas y sociólogos. Desde los años setenta empezó a cambiar la visión que se tenía de las organizaciones y se dejaron de considerar como una "caja negra". Los economistas empezaron a observarlas en su interior, lo que dio lugar a las teorías del principal y el agente y de los costos de transacción (Williamson 1975, 1981, 1985). Para esta última, las redes son maneras de reducir la incertidumbre, el riesgo y la ineficiencia. En el campo de la gerencia estratégica predomina la visión de la empresa basada en los recursos ${ }^{3}$, que la considera como una canasta de recursos y capacidades, y como creadora potencial de valor agregado, derivada de la concepción schumpeteriana de la creación de valor.

En el campo de la sociología, Podolny y Page (1998) introdujeron la noción de redes desde el punto de vista de la gobernanza ${ }^{4}$. Esta noción se suele usar para caracterizar formas específicas de organización basadas en relaciones sociales, la confianza y el uso común de recursos complementarios. Powell (1990) sostiene que la reciprocidad es un principio guía de la organización en red (cada miembro siente

${ }^{1} \mathrm{La}$ autora participó como becaria al inicio del Proyecto PICTO INTA n.o 02-12939: "Los acuerdos interempresariales como estrategia de supervivencia y expansión de las Pymes agroalimentarias" de la SECyT (octubre de 2004-marzo de 2005).

${ }^{2}$ Pasantía de la Universidad Nacional del Sur para jóvenes docentes con destino al Instituto de Economía, Universidad Estadual de Campinas (abril-julio de 2009).

${ }^{3}$ Ver Prahalad y Hamel (1990), Barney (1991), Liebeskind et al. (1996), Powell et al. (1996) y Dyer y Singh (1998).

${ }^{4}$ Para el Banco Mundial, gobernanza es el ejercicio de la autoridad política y el uso de recursos institucionales para administrar los problemas de la sociedad. Desde el punto de vista corporativo es la forma en que los directivos dirigen una empresa, y las costumbres y reglas que se aplican. 
obligación hacia los demás). Granovetter (1985) resalta los altos niveles de confianza y compromiso entre las partes.

Como ya se dijo, aquí se considera que una red es una agrupación de empresas y que estas tienen límites. Gran parte de la competitividad de las empresas se logra en las relaciones con otras empresas y con su entorno. Según Dini (2010), en una red existe la identificación positiva como grupo, se acuerda una estrategia común y se establece un mecanismo para tomar decisiones.

Existe una abundante bibliografía sobre tipologías de redes que usan criterios distintos. Con respecto a la tipología de acuerdos entre empresas, Castellano et al. (2005) presentan la siguiente clasificación: por tipo de actividades: producción, distribución, innovación, etc. ${ }^{5}$; por tipo de actores: grandes empresas y pymes, entre pymes, etc. ${ }^{6} ;$ por la amplitud del acuerdo: alianzas amplias o focalizadas ${ }^{7}$; por la modalidad del acuerdo: joint venture, franquicias, spin-off, subcontratación, etc. ${ }^{8}$.

Storper y Harrison (1991) clasifican los acuerdos según las relaciones de poder/dependencia entre las firmas: acuerdos basados en mecanismos de control/dependencia, de coordinación y de cooperación. Más en general, Perroux (1974) argumenta que los agentes no son aislados y que sus interacciones están marcadas por relaciones de poder. Las asimetrías de poder son inherentes a las relaciones sociales y económicas y, por tanto, a las redes de empresas, aun en redes menos asimétricas en cuanto al tamaño del capital de los participantes (Bacic e Souza, 2008).

Por su parte, Dini (2010) clasifica las redes de empresas según los siguientes criterios: número de participantes (bilateral, multilateral) y tipo de cadena productiva (horizontal, vertical) (Schmitz, 1999); apropiación de los beneficios (Dini et al., 2006; Schmitz, 1999), la cual diferencia las redes de las asociaciones; horizonte de planificación (mediano o largo plazo), el cual diferencia las redes de las coaliciones transitorias.

\section{TeOría DE CLUBES}

También existen distintas definiciones de club según las motivaciones que llevan a formarlo. Para dar una definición unificada, Sandler y Tschirhart (1980) lo definen como un grupo voluntario que obtiene

\footnotetext{
${ }^{5}$ Costa (1989), Montebugnoli y Schiattarella (1989), Mariti (1989) y Hermosilla y Solá (1990).

${ }^{6}$ Kosacoff (2000), Mariti (1989) y Welch (1991).

7 (Faulkner (1992) y Miles y Snow (1992).

8 Costa (1989), Mariti (1989), Hermosilla y Solá (1990), Faulkner (1992) y Miles y Snow (1992).
} 
beneficios mutuos por el uso compartido de los siguientes elementos: características de los miembros, bienes que proporcionan beneficios excluibles y costos de producción. Los bienes club cierran la brecha entre bienes privados, cuyo consumo es rival y su exclusión es costosa, y bienes públicos puros, cuyo consumo no es rival y su exclusión es poco probable. En el caso de los bienes club se puede excluir del consumo a quien no pague el precio, y los miembros participan voluntariamente si anticipan un beneficio neto (mayor que la comisión o el costo de ingreso) (cuadro 1).

La formación de clubes es una alternativa eficiente a la provisión no gubernamental de bienes cuasi-públicos. E1 modelo de Buchanan muestra que la provisión voluntaria no solo es posible sino que puede ser óptima (más eficiente que la provisión pública).

Cuadro1

Bienes club y bienes públicos

\begin{tabular}{|c|c|c|}
\hline Criterio & Bien club & Bien público puro \\
\hline Consumo & Rival & No rival \\
\hline Congestión & $\begin{array}{l}\text { Existe; depende positivamente del número de } \\
\text { miembros (medida de uso del club), y negati- } \\
\text { vamente del nivel de provisión (tamaño) }\end{array}$ & No existe \\
\hline Rivalidad & Surge de la congestión & No son rivales \\
\hline Exclusión & $\begin{array}{l}\text { Existe un costo de ingreso: cuota o pago; } \\
\text { permite la financiación privada }\end{array}$ & No son excluibles \\
\hline Partición & Existe un número de clubes & No hay partición \\
\hline
\end{tabular}

Fuente: elaboración propia.

A diferencia de los bienes públicos puros, los bienes club admiten un uso compartido que puede llevar a la congestión, es decir, a la reducción de los beneficios o de la calidad cuando cierto número de miembros usan el bien. Por ejemplo, cuanto más grande es la bodega central de una red de empresas de materiales de construcción y menor el número de miembros, menor es la congestión.

Los bienes club requieren un grupo exclusivo, que excluye a los no miembros. La mayoría de los bienes públicos locales que tienen características de club son suministrados por el sector público y se financian obligatoriamente con impuestos. Una alternativa es que los suministre una agrupación no gubernamental, como el club. El voluntarismo es una característica del club (no del bien club) y la pertenencia al club es voluntaria; las personas se pueden agrupar en clubes según sus preferencias (demanda de bienes). Así, el club es un mecanismo de revelación de preferencias que permite la financiación no obligatoria de bienes de consumo colectivo; y la exclusión permite financiarlos privadamente. 
La exclusión hace posible controlar el uso, cobrando una cuota o pago a los miembros y manteniendo afuera a los no miembros. La cuota permite internalizar la externalidad de congestión y, con ello, una asignación teóricamente más eficiente de los recursos. La posibilidad de exclusión implica que hay incentivos para revelar las preferencias. Por tanto, en principio no es necesaria la intervención del Estado para que el bien sea suministrado a la población. Las empresas de un consorcio de exportación de lácteos, que pagan una cuota de pertenencia, son las únicas que usan la oficina de ventas.

El número de clubes es un elemento importante: la partición de la población es lo que induce la competencia entre clubes. En términos de redes de empresas, esto significa que la población o el universo de empresas se pueden separar o desagregar en redes.

En suma, la teoría de clubes se basa en dos premisas básicas: a) la congestión exige limitar el tamaño del club y lo convierte en una variable endógena, en algunos casos determinado por peajes o precios de congestión; en algunos modelos, la decisión de uso determina el tamaño del club; b) las decisiones de pertenecer al club, de su tamaño y de provisión son interdependientes.

Los primeros trabajos sobre teoría de clubes fueron los de Olson (1965) y Buchanan (1965). De acuerdo con Olson, los clubes se forman con el fin de explotar economías de escala y para el uso común de bienes públicos; además, subraya la necesidad de clubes exclusivos que limiten el número de miembros o el tamaño del club para evitar la congestión. Buchanan supone, por simplicidad, miembros homogéneos que comparten por igual el bien y los costos asociados; el club no discrimina a ningún miembro y puede excluir sin costo alguno a los no miembros. El club de Buchanan es una organización descentralizada y voluntaria que opera sin costos de transacción. Entre los motivos que llevan a formar clubes, Sandler y Tschirhart (1980) mencionan la preferencia por la asociación (Schelling, 1969), la reducción de costos derivada de las economías de escala (McGuire, 1972) y la reducción de costos debida a la producción en equipo y al uso compartido de los recursos (Hillman, 1978).

\section{Extensiones del modelo de Buchanan}

Desde la publicación del artículo de Buchanan, la teoría de clubes se ha extendido para incluir la financiación del bien entre los miembros, la heterogeneidad de los miembros, los costos de transacción y los clubes multiproducto. Cornes y Sandler (1996) hacen una revisión de las extensiones de la teoría de clubes. McGuire (1974) presenta una 
variante del modelo de Buchanan que supone miembros homogéneos que comparten por igual los costos del bien club y tasas de uso fijas. Las empresas que participan en consorcios de exportación horizontal suelen ser homogéneas y compartir la oficina de ventas.

Berglas (1976) permite una tasa de uso variable (Sandler y Tschirhart, 1980). El precio del bien club depende de la tasa de uso. Cuanto mayor es la tasa de uso individual del bien mayor es el efecto de congestión. Un ejemplo es el de las empresas de la red textil Agivest que venden en la Feria Nacional de la Industria Textil. Para un tamaño dado del stand de la red, cuanto más tiempo permanezcan la empresas en la feria mayor es la congestión (por ello deben turnarse).

El modelo de Buchanan varía si los miembros son homogéneos o heterogéneos. Cuando son homogéneos, cada uno utiliza la misma cantidad del bien y, por tanto, las decisiones de uso y de pertenencia son una decisión discreta. Cuando son heterogéneos, las decisiones de provisión, uso y pertenencia son más complicadas debido a la agregación de preferencias distintas, y se espera que los costos de transacción sean mayores que en los clubes homogéneos. Por ejemplo, los clusters industriales tienen miembros más heterogéneos que las empresas de un consorcio de exportación. Cuando hay heterogeneidad, la función de congestión varía según el tipo de miembro. La importancia de la congestión no anónima o diferenciada es que ahora el pago por pertenecer al club difiere entre los miembros.

Hasta comienzos de los años ochenta los modelos de teoría de clubes no incluían costos de transacción. Cuando los miembros son idénticos, persiguen los mismos objetivos y no se requiere un control centralizado (una autoridad). Más recientemente ha surgido el interés por los efectos de la exclusión, el monitoreo y los costos de hacer cumplir los contratos en la estructura de los clubes. Por ejemplo, en los sistemas productivos locales (APL) de productos artesanales de tipo regional, la especificidad del lugar y la existencia de relaciones donde la territorialidad es clave generan costos de transacción.

Otras extensiones incluyen el número de productos que se deben incluir en un club $^{9}$. En los clubes de producto único, las economías de escala pueden reducir el costo medio y favorecer mayores niveles de provisión. En los clubes multiproducto, las economías de alcance, por subaditividad de costos, alientan la inclusión y el uso compartido de bienes club múltiples. Por ejemplo, un consorcio de exportación

\footnotetext{
9 Ver, p. ej., los modelos de Berglas (1982, 1984), Brueckner y Lee (1991), Sandler y Tschirhart (1993) y Wooders (1989).
}

Revista de Economía Institucional, vol. i7, n.o 32, primer semestre/2oi5, Pp. 3 i 7-348 
horizontal puede ofrecer distintos bienes club: oficina de ventas, marca regional, conocimiento, etc.

Una característica de los clubes multiproducto es la existencia de un costo común, que depende del conjunto de facilidades provistas, bien sean de administración, de infraestructura común (redes de comunicación e informáticas) o de un mecanismo de exclusión. Este costo común no se puede asignar arbitrariamente a cada facilidad.

\section{TEORÍA DE EQUIPOS}

Desde los trabajos de Vickrey (1961) y Mirrlees (1971), la investigación sobre diseño de mecanismos busca minimizar o eliminar el problema de información asimétrica. En los contratos en que hay azar moral se busca inducir la acción eficiente cuando no es observable. En principio, si el producto de un equipo es resultado de acciones de las partes (como la producción en conjunto de un bien o servicio), la eficiencia se puede inducir mediante contratos contingentes a nivel de producto. El azar moral en un equipo surge porque no se puede observar el grado de esfuerzo de cada cual (su productividad o su nivel de producción). Hay azar moral aunque el nivel de producto del equipo sea observable. El diseño de mecanismos intenta minimizar las rentas de quienes tienen información privada.

Las organizaciones con muchos agentes tienen al menos dos características: a) producción conjunta, en la que surge el problema del gorrón (free riding, por qué esforzarme como miembro de un consorcio de exportación para producir en gran escala si el volumen exportado depende de todo el consorcio); b) competencia entre agentes para controlar los incentivos. Si el nivel de esfuerzo fuese observable (no existiría azar moral), el problema del gorrón se podría evitar con un sistema de incentivos (premios y sanciones), sin necesidad de un principal. El problema del gorrón no solo surge de la incertidumbre (derivada del azar moral) sino también de la búsqueda de equilibrio presupuestal (en un presupuesto balanceado la suma de las participaciones es 1). Los contratos que reparten equilibradamente el producto no motivan a los agentes neutrales al riesgo a hacer el esfuerzo óptimo (si las ganancias de una cooperativa se reparten equitativamente aunque los aportes individuales sean diferentes). Los agentes elegirán esforzarse poco, pues el costo de ese comportamiento será compartido entre todos los miembros mientras que los beneficios se atribuyen al agente individual.

Para resolver el problema del azar moral en equipos Holmstrom (1982) propuso romper el equilibrio presupuestal imponiendo san- 
ciones, y así neutralizar las externalidades derivadas de la producción conjunta. Otra solución es disciplinar el equipo mediante un contrato principal-agente (entre un coordinador y cada empresa) que redistribuye el producto del equipo a un tercero (el principal) si el producto no llega al nivel óptimo. Cada agente neutral al riesgo elige el nivel de esfuerzo óptimo. El principal capta el residuo derivado del quiebre del equilibrio presupuestal, y hay un trade off entre eficiencia y equilibrio presupuestal. Eswaran y Kotwal (1984) cuestionaron esta propuesta de un contrato principal-agente con sanciones porque puede generar un nuevo problema de azar moral si el principal hace contratos paralelos con uno o varios miembros del equipo para que el nivel de producto sea inferior al óptimo.

Por su parte, Rasmussen (1987) argumenta que en condiciones de incertidumbre los incentivos son efectivos si el número de agentes tiende a infinito y los agentes son adversos al riesgo, pues los contratos que distribuyen equilibradamente el producto sí incentivan el esfuerzo óptimo. Así, el problema de azar moral se puede resolver sin un principal o limitando su tarea a la supervisión. Con aversión al riesgo la sanción puede tomar la forma de una lotería. Si los agentes tienen suficiente aversión al riesgo o la lotería es suficientemente riesgosa, ningún agente desea desviarse del nivel eficiente de esfuerzo pues cree que los demás harán el nivel de esfuerzo óptimo. Rasmussen prueba, además, que los agentes no tienen incentivos para evitar el esfuerzo óptimo cuando la diferencia entre hacer ese esfuerzo y mentir es positiva. En estos contratos se procura disminuir la utilidad de todos los agentes una vez se observan ciertos productos, y en ellos se debe prevenir la renegociación.

En resumen, Holmstrom y Rasmussen distinguen dos clases de contratos: un contrato de todos los agentes (entre las empresas de una red) y un contrato principal-agente (entre el coordinador de la red y cada empresa); el primero solo involucra a los miembros del equipo y el segundo introduce una tercera parte (el principal) en el contrato. El principal no tiene un impacto directo en el nivel de producto del equipo, es decir, nada agrega al proceso de producción.

\section{Ampliaciones del modelo de Holmstrom}

Además del problema de azar moral, McAfee y McMillan (1981) introducen el de selección adversa, suponiendo que la habilidad y el esfuerzo interactúan de tal modo que el principal no puede separar el esfuerzo del agente de su habilidad. 
Suponiendo que el principal y los miembros del equipo son neutrales al riesgo y que solo es observable el producto del equipo, el contrato óptimo especifica hacer a cada agente un pago lineal sobre el producto total del equipo. Este contrato no es óptimo, todas las empresas -salvo las de costo de producción más bajo- producirán un nivel inferior al óptimo, y todas -salvo las de mayor costo de producción- obtendrán rentas positivas. Si el principal busca extraer esta renta a las empresas, estas reducirán su productividad. De este modo, el principal enfrenta la opción de extraer rentas a las empresas o inducirlas al nivel de productividad óptimo. La utilidad esperada para el principal es igual bien sea que observe el producto total o que supervise la contribución de cada empresa; es decir, el principal no obtiene beneficios por supervisar.

Según Vander Veen (1995), si los agentes son adversos al riesgo y el principal puede supervisar sin costo las contribuciones individuales, este puede encontrar una nueva regla de distribución del producto que paga a las empresas menos que su contribución pero las mantiene en el mismo nivel de utilidad. Con igual contribución al producto y menor pago a las empresas, el principal logra aumentar la utilidad esperada. El pago ahora es no lineal (a diferencia del modelo anterior). Con la supervisión, el principal puede eliminar la prima de riesgo que debe pagar a las empresas adversas al riesgo (la menor incertidumbre tiene un costo para la empresa).

Por su parte, Andolfatto y Nosal (1997) consideran que si el principal tuviera acceso a una riqueza “exógena” se podría impedir la asignación óptima en el contexto de Holmstrom. Pues surge la posibilidad de negociaciones clandestinas que impidan la realización del contrato principal-agente. Sin embargo, si se hace un pago al principal, aun en el caso de alta producción, sus incentivos para sobornar a las empresas y hacer que mientan pueden reducirse.

Hasta ahora la teoría de los incentivos se ha basado ante todo en las relaciones de corto plazo entre empresas y ha abogado por el uso de esquemas de incentivos que sean sensibles a las medidas de desempeño individual e induzcan la competencia entre empresas mediante premios o la evaluación del desempeño relativo (Hart y Holmstrom, 1987). Aunque estos modelos estáticos llevaron a muchos descubrimientos, omiten el hecho de que los sistemas de incentivos actuales son de bajo poder y rara vez involucran la evaluación del desempeño relativo.

En el análisis de los incentivos Che y Yoo (2001) introducen posibles interacciones de largo plazo entre las empresas, y estudian 
cómo interactúa el diseño de incentivos explícitos y de la organización del trabajo en red con los incentivos implícitos generados por la interacción repetida de las empresas, y cómo se puede explotar. El esquema de incentivos óptimo tiene características de los equipos. Si bien las características de los equipos exitosos varían según los casos, su filosofía común consiste en fomentar la cooperación entre empresas mediante varias particularidades de las prácticas de trabajo.

1.Los miembros de equipos auto-organizados interactúan frecuente y consistentemente durante un periodo (interacción de largo plazo).

2. Se estimula a los miembros a tomar decisiones diarias sobre la asignación del proyecto y la solución de problemas (autoridad descentralizada).

3. En vez de tener supervisión externa, se impulsa a los miembros del equipo a supervisarse y motivarse entre sí (supervisión de pares).

4. En vez de un esquema competitivo como la evaluación del desempeño relativo, los miembros del equipo trabajan con esquemas de incentivos que mejoran la cooperación, como los incentivos de grupos (evaluación del desempeño conjunto).

Che y Yoo (2001) analizan los problemas de cumplimiento de los contratos asociados a la cooperación introduciendo interacciones repetidas, de largo plazo. Estas interacciones pueden crear incentivos implícitos que impulsan a las empresas a imponer sanciones entre pares (afectar su reputación). El diseño de incentivos explícitos, mediante esquemas de compensación y asignación de tareas, influye en el carácter y el alcance de la interacción dinámica y, por tanto, en el poder de los incentivos implícitos. Su modelo indica que las organizaciones que tienen larga vida y llevan contabilidad suelen tener incentivos de grupo de bajo poder, y que las organizaciones de corta vida o sin contabilidad tienen esquemas de incentivos competitivos de alto poder, como la evaluación del desempeño relativo.

Señalan, además, que las prácticas de trabajo que funcionan bien en organizaciones de corta vida pueden no ser buenas para las organizaciones de larga vida. En particular, aquellas que promueven la competencia o separan los intereses de las empresas pueden no funcionar bien cuando las empresas interactúan repetidamente. Este escenario requiere el uso de prácticas que entrelacen el destino de las empresas, porque aumentan el poder de los incentivos implícitos (como el sistema de inventarios justo a tiempo) que crean más dependencia entre las empresas y ayudan a formar lazos sociales entre ellas (capital social). 


\section{CASOS DE REDES O ALIANZAS ENTRE PYMES}

La mayoría de los casos de cooperación no son ejemplos "puros" de clubes o equipos ${ }^{10}$; no obstante, aquí se analizan algunos casos de redes de Argentina y Brasil y se los enmarca en una de esas dos teorías, la que los caracterice mejor, la cual depende de que el principal objetivo de la red sea el trabajo en equipo para lograr un fin o realizar un emprendimiento colectivo (equipo) mientras que las empresas participantes persiguen su interés individual; o de que las empresas de la red absorban las externalidades de un bien club o compartan ciertos recursos comunes y exclusivos a la red. En algunos casos, como los clusters o distritos industriales, las empresas comparten algún bien club -información, experiencia, proveedores comunes, etc.- y al mismo tiempo tienen las dimensiones de un equipo. Por ello se pueden interpretar fácilmente desde ambas teorías (clubes y equipos).

Mediante la teoría de clubes se examinan casos de consorcios de exportación horizontales y cooperativas agropecuarias. Y mediante la teoría de equipos, consorcios de exportación verticales y redes de empresas brasileñas de materiales de construcción, panaderías, empresas textiles, farmacias y supermercados. La información de las redes entre empresas se obtuvo de fuentes de información secundaria y primaria.

\section{Clubes}

\section{Consorcios de exportación horizontales}

Según la Organización de las Naciones Unidas para el Desarrollo Industrial (UNIDO), un consorcio de exportación es una alianza voluntaria entre empresas con el fin de promover las exportaciones de bienes y servicios a través de acciones conjuntas de sus miembros.

Como las firmas se juntan para vender al extranjero, comparten recursos comunes - p. ej., una oficina de ventas- que generan beneficios debido a la subaditividad de costos; tienen entonces características de un club.

Las empresas que participan en un consorcio comparten información, experiencia, conocimiento y aprendizaje. El conocimiento tácito y la experiencia solo fluyen entre ellas y no tienen el carácter de bien público. Gran parte del conocimiento, sobre todo el know-how (destrezas y habilidades) y el know-who (redes sociales, interacciones personales y capital social), está incorporado en las personas y no es codificado; por tanto, es costoso difundirlo y aplicarlo en otros

\footnotetext{
${ }^{10}$ Ejemplo "puro" de club o de equipo alude a la idea de una red que reúne las características de un equipo, o de un club, pero no de ambos casos.
} 
contextos. Si bien el elemento codificado es transable, el elemento tácito es específico de la red; no se puede comprar en el mercado y es un elemento clave de las diferencias tecnológicas y las ventajas competitivas específicas de las redes. Este tipo de conocimiento se puede considerar un bien club (Cornes y Sandler, 1996), y podemos distinguir entre miembros del club y agentes externos. También se puede considerar que el aprendizaje colectivo que se transmite entre los miembros de un consorcio es un bien club, pues no hay rivalidad entre las empresas del consorcio que lo usan y excluye a las empresas externas, que no pueden aprovechar esta externalidad.

En Argentina varios autores han detectado una limitada orientación exportadora de las pymes ${ }^{11}$, aunque hay pocos estudios empíricos por falta de bases de datos apropiadas (una excepción es la base de datos del Proyecto PICTO INTA n.o 02-12939). Castellano et al. (2005) describen y analizan el papel del coordinador en la formación y el funcionamiento de grupos de exportación (GE) compuestos por pymes agroalimentarias nacionales; para ello elaboraron una base de datos con el listado de los GE de pymes agroalimentarias.

Entre ellas se observan distintas formas de asociación para exportar, desde cooperativas, consorcios y clubes de exportación hasta sociedades. La industria alimenticia es un ejemplo de cooperación en el que las empresas comparten información y experiencia. Lahore SA y Modesto Bertolini SA, participantes en el nuevo modelo exportador de la industria lechera, unieron esfuerzos para exportar 12 toneladas de queso duro reggiano a Estados Unidos en 2005. Su relación comenzó a través de Apymel (Asociación de Pymes Lecheras), de la que eran miembros, y del consorcio de exportación Argendairy, creado por pymes que exportan queso suave de cabra y oveja ${ }^{12}$. A la red para exportar queso, que nació a partir de objetivos comunes y de una relación comercial y personal, se han unido empresas pequeñas y medianas debido a que una sola empresa no puede satisfacer los altos volúmenes de exportación requeridos. Entre los productores de queso de variedad, el consorcio puede hacerse cargo de la compra de leche, de la marca del producto para grandes lotes, de la exportación, de la marca regional y de la garantía de calidad. Los participantes pueden pactar que los pedidos sean atendidos por cada empresa con su propias marcas hasta cierto volumen, y que ventas por encima de ese volumen sean atendidos por el consorcio, con su marca y garantía de calidad.

${ }^{11}$ Ver Kantis (1994), Yoguel y Boscherini (1996) y Alderete (2007).

${ }^{12}$ Ver Mateos et al. (2009), que analizan la situación de las pymes que elaboran quesos en la provincia de Buenos Aires.

Revista de Economía Institucional, vol. i7, N. ${ }^{\circ} 32$, Primer semestre/2oi5, Pp. 3i7-348 
Por otra parte, según el Informe de Actividades del Programa Bank Boston Export-Ar de 2005 hay 41 grupos exportadores, en los que participan cerca de 300 empresas y exportan 117 millones de dólares. Estos grupos exportan diversos productos: muebles, autopartes, equipo médico, alimentos envasados, alimentos gourmet y uvas pasas. Desde el comienzo de este programa ha aumentado el número de grupos y de firmas: en 1998 solo había 3 grupos formados por 30 empresas y en 2001 ascendían a 25 grupos y 214 firmas. Aquí podemos identificar los siguientes ejemplos de clubes (empresas que producen un bien homogéneo):

- Grupo Exportador de Uvas Pasas, integrado por 7 empresas, que inició actividades dentro del Programa de Bank Boston-Exportar, en noviembre de 2001.

- Grupo Exportador de Orgánicos (Conexport), formado por 6 empresas, que inició sus actividades dentro del programa en mayo de 2003. Su producto más destacado es el jugo de uva concentrado o mosto.

- Grupo Exportador de Frutas Frescas (SJ Fruits), compuesto por 7 empresas, que inició sus actividades en octubre de 2001. Su principal producto de exportación es la uva fresca de mesa.

\section{Las cooperativas agropecuarias}

En Argentina, la principal experiencia asociativa es la de las cooperativas agropecuarias, que promueven la integración entre las industrias y los productores con o sin colaboración del Estado (SAGPyA e INTA), y donde se forman grupos para comprar en forma conjunta insumos (semillas y agroquímicos) o vender sus productos a mejores precios. En este sentido actúan como un equipo. La asociación permite acceder a capacitación y asistencia técnica y usar de modo eficiente las tecnologías existentes, actuando a la manera de un club. En el sector agropecuario el cooperativismo tiene mayor antigüedad: los pequeños productores comparten tractores y cosechadoras.

\section{EQUIPOS}

\section{Consorcios de exportación verticales}

En este caso, cada miembro aporta una parte al producto final. Ejemplo de ello son los consorcios para equipamiento odontológico o fabricación de móviles, donde la producción de los componentes se distribuye en cadena entre los miembros, y el montaje final puede estar a cargo de las empresas del consorcio o de una firma específica. 
El consorcio de exportación Components \& Machinery de Brasil, creado en 2003, fue resultado de la unión de los principales fabricantes de cuero, máquinas y componentes para fabricar calzado. Este consorcio facilita la compra de insumos y ofrece calidad internacional y precios competitivos. Nació como una iniciativa de la Asociación Brasilera de Empresas de Componentes para Cuero, Calzado y Artefactos (Assintecal), a través de la cual recibe aportes financieros de la Agencia Brasileña de Promoción de Exportaciones e Inversiones (APEX). Hoy participan 14 empresas.

Cabe destacar que las empresas del consorcio no compiten entre sí, un prerrequisito que establece el contrato. Buscan, en cambio, formar una cadena productiva de productos complementarios y no de un mismo tipo. Las empresas integrantes que ya exportaban aumentaron sus exportaciones entre un $10 \%$ y un $15 \%$. Si los productos no son de alta calidad las empresas no son aceptadas en el consorcio. Las firmas integrantes exportan sus productos con marca propia, mientras que la marca comercial del consorcio Components \& Machinery de Brasil se usa en catálogos, ferias internacionales y en su página web.

\section{Uniones transitorias de empresas}

En Bahía Blanca, las iniciativas asociativas en que participan las pymes son básicamente uniones transitorias de empresas (UTE). Una UTE consiste en un contrato plurilateral mediante el cual las firmas se unen entre sí o con empresarios individuales para ejecutar una obra, proporcionar servicios o suministros concretos y realizar obras o prestar servicios complementarios al objetivo principal (art. 377 de la Ley de Sociedades de la República Argentina, Ley 19.550). Las firmas en UTE actúan como un equipo, prestan servicios a terceros y pueden tener fines de lucro, al menos indirectamente. No tienen la característica de equipos que interactúan frecuentemente y consistentemente durante un periodo, pues su interacción tiene una duración igual a la de la obra, servicio o suministro.

\section{Clubes y eQuitos}

\section{Clusters o distritos industriales}

Los clusters, también conocidos como distritos industriales, son conglomerados de empresas y entidades de la misma cadena productiva en los que hay cierta especialización. La UNIDO define los clusters como concentraciones sectoriales y geográficas de empresas que producen $\mathrm{y}$ venden productos relacionados o complementarios $\mathrm{y}$, por tanto, 
enfrentan retos y oportunidades comunes. La aglomeración es ante todo sectorial. Por ejemplo, los clusters del calzado, del vino, de lácteos, etc. Esta característica convierte a las empresas de un cluster en un equipo, pues participan en la producción de un bien dado. Dentro de un cluster pueden existir varias redes y consorcios empresariales. Las redes de empresas del cluster que producen cada componente o que participan en cada etapa de la cadena (las etapas verticales pueden ser producción, procesamiento, exportación) comparten a la vez las características de un club.

En su condición de clubes, los clusters o distritos brindan a las empresas participantes una ubicación o sede propia. Así, las pymes se benefician y aprovechan las condiciones de la economía local (infraestructura, mano de obra especializada, recursos naturales locales, etc.). Solo las empresas del cluster pueden aprovechar la infraestructura local del distrito y la mano de obra especializada, lo que les da características de un club. Puede haber congestión si el número de empresas localizadas en el mismo cluster reduce los beneficios derivados del uso de estos recursos comunes.

En Brasil, los acuerdos productivos locales (APL) son aglomeraciones territoriales de agentes económicos, políticos y sociales ligados a conjuntos específicos de actividades productivas vinculadas entre sí. Surgieron con la nueva política industrial de mediados de los noventa que adoptó medidas para fomentar la innovación y el acceso de las pymes a los mercados interno y externo. Tuvieron el apoyo de instituciones como el Servicio Brasileño de Apoyo a la Pequeña Empresa (Sebrae), y entre ellos cabe mencionar a Nova Friburgo (moda íntima), Tobias Barreto (confecciones y bordados), Campina Grande (cuero y calzados) y Paragominas (muebles).

En Argentina, el desarrollo económico, industrial, social e institucional ha impedido la creación de agrupaciones de empresas en clusters o distritos. Según Boscherini y Poma (2000), ese proceso ha sido limitado o impedido por dos razones principales: a) la falta de capacidad empresarial independiente y una actitud cooperativa de las empresas y, en sentido más amplio, de los agentes económicos e institucionales; b) un sistema institucional que no acompaña y fomenta las actividades del sector económico y de la sociedad civil y, en particular, que no estimula y apoya los mecanismos asociativos, como sí se hace en Brasil. En este contexto, las pymes tienen poca capacidad empresarial y poca autonomía, su actitud es individualista y poco dinámica, etc.

No obstante, algunas entidades fomentan la articulación entre empresas. E1 Instituto de Desarrollo Empresario Bonaerense 
(IDEB) es una entidad no gubernamental que involucra y relaciona a los actores socioeconómicos locales que trabajan para desarrollar $\mathrm{y}$ fortalecer las pymes de la región. Su programa de promoción de clusters y redes productivas da apoyo técnico y financiero a las mipymes que forman bloques productivos (grupos asociativos de 5 o más empresas) o adoptan otros formas de articulación vertical u horizontal.

Pero hay casos de actividades que tienen características de cluster, como el Rafaela, un cuasi distrito industrial que agrupa empresarios metalmecánicos de pequeña escala, en el que tienen gran peso las pymes que producen frigoríficos (25\%), lácteos (17\%), curtiembres (16\%) y propiamente metalmecánicas (más del 20\%). El sistema de Rafaela, ubicado a más de $500 \mathrm{~km}$ del Polo Metropolitano, es un caso especial de desarrollo manufacturero fuera de las áreas industriales tradicionales de Argentina. Su buen desempeño en los últimos años ha sido favorecido por el dinamismo y el protagonismo de las instituciones y agentes del entorno próximo del cuasi-distrito.

Otro ejemplo de cluster productivo es el de la producción de vino, predominante en Mendoza, San Juan y La Rioja. Igual que otros complejos agroalimentarios del país, este muestra, desde los años noventa, un fuerte dinamismo, especialmente en el segmento de vinos finos de mesa, con grandes aumentos de la producción y la productividad, notables innovaciones tecnológicas en la producción primaria, estrategias de diversificación de productos y marcas, segmentación de mercados y una fuerte reorientación exportadora.

Al mismo tiempo, hay una fuerte tendencia a la heterogeneidad en la producción primaria y al desplazamiento de pequeños viñateros. En Mendoza la producción primaria está atomizada. E1 96\% de los 18.315 viñedos existentes en 2001 tenía menos del 25\% y pertenecía a unos 12.000 viticultores. Un $40 \%$ está asociado a cooperativas (de la Federación de Cooperativas Vitivinícolas, Fecovita), un 15\% tiene contratos más o menos formales con bodegas, y los demás son productores heterogéneos con gran dispersión espacial. Los productores primarios no integrados venden la uva (en especial uva fina) a las bodegas, la uva de mesa la venden a las bodegas integradas o la entregan "trasladistas" y estas la "elaboran por cuenta de terceros"(maquila). En ambos segmentos hay cooperativas de productores con producción integrada. Por su parte, las políticas orientadas a la vitivinicultura y sus exportaciones promueven la formación de asociaciones de empresas y la creación de nuevos organismos públicos no estatales, con participación del estado provincial. 


\section{REDES DE EMPRESAS EN BRASIL}

\section{Redes de materiales de construcción y panaderías}

En Brasil, la principal actividad en la que se han formado redes es la panadería, seguida de la venta de materiales de construcción. Estos casos se asemejan a consorcios de compras, que consisten en la cooperación horizontal de firmas independientes que reúnen la compra de insumos para obtener beneficios como menores costos por reducción de precios, menores costos de administración, menores costos logísticos y flexibilidad de los inventarios.

Cuadro 2

Panaderías y materiales de construcción, Brasil

\begin{tabular}{lll}
\hline Características & Materiales de construcción & Panaderías \\
\hline Reducción de costos & $16 \%$ & $12 \%$ \\
Redes activas & Muchas & Pocas \\
Costos de salida & Altos & Bajos \\
Estabilidad & Estable & Inestable \\
Competencia & Cooperan en la compra de insumos & \\
Otros beneficios & Compiten en la comercialización & \\
Ejemplos & Cursos y capacitación a empleados & Grupos Panifica- \\
& Red Construir, Construvip, Constru- & dores SP Grupan, \\
\hline
\end{tabular}

Fuente: elaboración propia.

El cuadro 2 muestra que la reducción promedio de los precios por la compra en red fue del $12 \%$ en las panaderías y del $16 \%$ en la venta de materiales de construcción, como era de esperar dado que el principal motivo de su formación era aumentar el poder de negociación en la compra de insumos. Estas redes de empresas independientes dan una señal de entidad compacta a los contratistas, exploran en conjunto los mercados oferentes, preparan contratos y deciden el procedimiento de reclamo. En este sentido, trabajan como equipos. Por otra parte, debido al mayor volumen de compras, los vendedores de material de construcción deben ampliar el espacio destinado a almacenamiento y crear una central unificada, lo que lleva a integrar los programas de computación y las actividades de logística, y baja los costos operativos (un efecto red positivo [Shapiro y Varian, 1998] con altos costos de salida). En este sentido operan como $c l u b$, ya que las empresas que no componen la red no tienen acceso a la central de almacenamiento.

Las redes llevaron a mejorar la calidad y la atención de los proveedores. Sus miembros consideran que la calidad de los productos comprados en red es mejor debido al mayor volumen de compras, aunque deben demandar el mismo tipo de insumos o bienes, pues para 
que la red opere eficazmente los bienes comprados deben tener alto grado de estandarización. A veces no solo deben dar apoyo financiero sino asumir el compromiso de asignar recursos humanos para que las operaciones sean bien gestionadas. $\mathrm{Y}$ se comprometen a comprar a los proveedores escogidos por el consorcio.

Estos elementos no se encuentran en las redes de panaderías de compras conjuntas. Como cada miembro siempre compara el precio colectivo de la compra de insumos con el precio que consigue por aparte (de proveedores no escogidos por la red), la red es inestable y sujeta a comportamientos oportunistas. Como no hay un fuerte compromiso para mantenerse en la red y el costo de salida es bajo (además no existen sanciones por retirarse), lo máximo que puede perder es el descuento en el precio de compra de un insumo.

Según Bacic y Souza (2008), las redes que exigen alto compromiso inicial de los participantes, dada la necesidad de integrar las acciones para formarlas, tienden a sobrevivir más que las redes constituidas con poco compromiso. Los altos costos de salida están vinculados a la especificidad de los activos (Williamson, 1985). Una vez se comprometen recursos en un activo específico (p. ej., inversión en locales de venta de materiales de construcción) es difícil o imposible reutilizarlos al cesar esa actividad.

La red de panaderías Sindipan, de los panificadores de San $\mathrm{Pa}$ blo, lanzó como equipo un nuevo producto, el pan francés light. Para mantener la calidad del producto da entrenamiento en las áreas de atención, higiene y limpieza, y así capacita a las empresas asociadas. Si una empresa falla en la calidad afecta al resto y desmejora la reputación del grupo. Por su parte, la red Croco Pão surgió como central de compras (club) y luego incorporó productos diferenciados (equipo). Uno de sus objetivos es mejorar la calidad con programas de entrenamiento o capacitación para los asociados y empleados de las panaderías.

\section{Red Agivest}

Un problema que suelen enfrentar las pymes cuando compiten en los mercados es el bajo volumen de producción. Balestrin y Vargas (2004) estudiaron la red Agivest (Asociación Gaucha de Industria de Vestimenta) formada por pymes localizadas en el sur de Brasil. Esta red se creó en 2001 con el fin de ampliar el mercado, hacer mejoras tecnológicas y lograr mayor competitividad para las empresas asociadas. El problema de la baja escala se atenuó notablemente debido a que la capacidad de producción se multiplicó al actuar en red. Así, 
Agivest logra economías de escala (por el volumen de producción) y de especialización (cada empresa produce algunas piezas que forman parte de la colección o pedido de un cliente). En cuanto busca cierto volumen de producción para alcanzar una escala de venta de un producto, Agivest actúa como un club; y los miembros deben producir las piezas en la cantidad y con la calidad acordadas (lo más homogéneas posibles). Las empresas dedicadas a la confección trabajan con dos marcas: la marca de cada empresa para los productos que comercializa individualmente y la marca Agivest para los que comercializa la red. Tienen entonces características de club. Cuando trabajan en forma coordinada para fabricar y unir las piezas de una colección o pedido, actúan como equipo.

Esta red está formada por 18 pequeñas empresas, con un número promedio de 6 empleados, ubicadas en un radio de $180 \mathrm{~km}$. Entre los beneficios que han obtenido por actuar en red, se destaca el intercambio de información y de conocimientos, sobre todo en materia de procesos de producción, proveedores, insumos, tecnologías y mercados. Esta información se transmite a través de la intensa interrelación formal e informal entre los empresarios. Cada empresa aprovecha el conocimiento y la experiencia de las demás.

Agivest también proporciona otros beneficios a través de la participación en la Feria Nacional de la Industria Textil, el cabildeo con los gobiernos municipal y estatal para obtener incentivos, la mejora de los procesos (la consultoría contratada por la red llevó a modificar algunos procesos productivos y la producción se incrementó en un $40 \%$ sin aumentar el personal ni comprar maquinaria), los cursos de capacitación, las transacciones con proveedores (antes se compraba a mayoristas y ahora directamente a las fábricas, con una reducción de costos cercana al 25\%, el mercadeo conjunto y la garantía de créditos (p. ej.,Agivest contrató una consultora de crédito para prestar servicios a todas las empresas asociadas).

Agivest ha establecido estrechas relaciones con entidades comprometidas con el desarrollo de las pymes de la región: los gobiernos municipales y estatales (por medio de SEDAI), las universidades (Universidad de Ijuí) y el Sebrae.

\section{Redes de farmacias}

En el Estado de São Paulo, algunas farmacias se unieron en una asociación de farmacias en 1997, para hacer compras conjuntas. Hoy, su propósito no se limita a la compra, se extendió a la venta y llegada al consumidor final con una marca propia. Es decir, se pasó de ser 
un club (pertenecer a la red de compras de medicamentos) a ser un club-equipo, con el lanzamiento de un producto con marca propia.

Para que las droguerías entren a la asociación no deben tener deudas, deben cumplir el código de ética definido por la red y modificar el esquema comercial conforme a sus normas de presentación. Así, el costo de entrar en la red depende de las modificaciones que haya que hacer para cumplir los requisitos. Las droguerías tienen flexibilidad para salir de la red, y la decisión se aprueba en asamblea. Además, su facturación mínima mensual debe ser de 30 mil reales. En el caso de Farma Vip se procuró excluir a las droguerías que no agregaban valor y centrarse en las que se consideraban eficientes. Farmáxima, por su parte, no solo exige una facturación mínima a los socios sino, además, un tiempo mínimo de dos años en el mercado. Net Farma hace hincapié en la necesidad de que los asociados inviertan para poder competir. Las droguerías asociadas deben cumplir un estándar de imagen externa e interna.

\section{Un caso de integración horizontal}

Febrafar, Federación Brasilera de las Redes Asociativas de Farmacias, es una red de redes. Se fundó en febrero de 2000 como sociedad civil sin ánimo de lucro, con sede en São Paulo y operación en las principales ciudades de Brasil. Hoy congrega 26 redes de farmacias independientes en 11 estados, además del Distrito Federal. Las 2.500 farmacias de sus redes asociativas operan en más de 900 municipios y facturan al mes cerca de 80 mil reales. El número de integrantes de cada red varía, de 20 a 400, y su carácter es heterogéneo. Su presidente señaló que los vínculos son más estrechos y frecuentes en las redes más pequeñas que en las más grandes, las cuales establecen un par de objetivos y luego funcionan con gran independencia.

El objetivo de Febrafar es integrar y fortalecer las redes asociadas, mejorar la representación política de sus afiliados y contribuir al desarrollo social y económico. Entre los productos que comercializan las farmacias asociadas, los medicamentos representan cerca del $65 \%$; el 35\% restante corresponde a productos de higiene personal, perfumería, cosmética, etc. Febrafar cumple las siguientes funciones: sistematizar información y unificar las redes asociadas; informar a los asociados sobre la realidad económica, tecnológica y política del sector, y trazar estrategias acordes con los cambios del mercado; contribuir a la expansión y divulgación de los servicios de cada red, y sobre todo, al éxito conjunto; organizar cursos de actualización y capacitación profesional a los empleados y colaboradores de las redes asociadas; 
promover congresos, simposios, ferias para estrechar las relaciones de la cadena farmacéutica.

Las tecnologías de la información y de la comunicación son esenciales para administrar la red. Las redes y la federación usan un sistema de software de administración integrado. Este sistema permite hacer consultas sobre cada asociado, sobre los proveedores, sobre los pedidos (marca, volumen, precios) y otras más. Cada farmacia hace sus pedidos usando el sistema y los atienden los proveedores que disponen de él, los cuales hacen descuentos en los precios de los productos por formar parte de la red.

Febrafar contrata con terceros el diseño de los sistemas informáticos SIC Febrafar y Panel de gestión do Varejo, pero la red está a cargo de su administración. El panel de gestión calcula diversos indicadores de desempeño de las farmacias. La red Febrafar es similar a un sistema de franquicia pero difiere en que las reglas del negocio son establecidas por la federación y no impuestas por una casa matriz.

\section{Redes de supermercados}

Las redes de supermercados exigen algunos requisitos para formar parte de la red de comercio minorista, como un número mínimo de cajas y de metros cuadrados de superficie, demostrar idoneidad, y tener credibilidad con los proveedores y pagar una tasa de ingreso, más una mensualidad. En algunos casos exigen algunos años de antigüedad en el negocio. Entre las principales redes se encuentran: Super Vizinho, Super Mais, Central de Compras, Associação Londrinense de Empresários Supermercadistas (ALES), Associação dos Varejistas do Estado do Rio de Janeiro (AVERJ), Unisuper, União Gaúcha de Supermercados y Rede Super.

Super Vizinho es un grupo de 47 supermercados creado en 2001, que opera en el área de Grande São Paulo, mediante una alianza con el Sindicato del Comercio Minorista de Géneros Alimenticios del Estado de São Paulo (Sincovaga) y la FIA-USP. La estrategia de Super Vizinho es formar alianzas de supermercados para obtener ganancias en todas las etapas de los procesos comerciales y operativos. Para formar parte de esta red el supermercado debe tener al menos cinco cajas y 300 metros cuadrados de superficie; la tasa de ingreso es de 100 reales y la mensualidad de 500 reales. A cambio se beneficia por la compra en grupo, la propaganda compartida, la capacitación del personal, la asesoría comercial, la tarjeta de crédito común y el intercambio continuo de información. 
En la red de supermercados AVERJ se pagan una tasa de admisión y aportes mensuales fijados por el directorio. Los miembros potenciales deben presentar una propuesta con toda la información requerida para ser admitidos. Hoy se pagan 25 salarios mínimos como cuota de entrada, pagaderos en 5 cuotas. La solicitud de ingreso como miembro debe ser analizada por tres proveedores de la zona respectiva seleccionados por AVERJ, y además el solicitante debe ser presentado por un socio de AVERJ.

Por su parte, la red Unisuper no se limita al sector de supermercados (donde actúa como un club), sino que es un canal para vender productos de la marca "Sabor Gaucho", que cobija 11 agroindustrias del interior de Rio Grande do Sul: conservas, embutidos, harinas, granos etc., producidos por 103 familias de agricultores.

\section{LAS REDES SEGÚN LAS TEORÍAS DE CLUB Y DE EQUIPO}

Esta sección muestra que los casos descritos se pueden interpretar como clubes y equipos de acuerdo con las dimensiones de ambas teorías. El análisis sería incompleto si solo se tomara en cuenta una de esas teorías, pues son complementarias para explicar la formación y el funcionamiento real de las redes de empresas. Los casos escogidos captan la heterogeneidad de las redes existentes, y no pretenden cubrir todas las redes y combinaciones posibles. Por ello los cuadros que aquí se presentan solo incluyen redes de producción, exportación, de venta de materiales de construcción, uniones transitorias de empresas, clusters regionales y acuerdos productivos locales.

A continuación se sintetizan las dimensiones que se tienen en cuenta en las teorías de clubes y de equipos, y se determina si las redes escogidas cumplen esas dimensiones y en qué medida. Es decir, se presenta la taxonomía de los casos que se describieron en las secciones anteriores. Luego se hacen recomendaciones sobre el mecanismo más adecuado para el buen funcionamiento de las redes.

Dimensiones de la teoría de clubes

1. Tipo de pertenencia.

2. Nivel de homogeneidad de los miembros, del producto ofrecido, etc.

3. Congestión. La red es o no susceptible de congestión (número de miembros, volumen de provisión del bien club).

4. Exclusión. Existe o no un costo de ingreso, pago o cuota, y otros mecanismos de exclusión.

5. Número de clubes. Existen otras redes en el sector (al menos con 2 miembros). 
6. Economías de escala. Existen o no.

7. Multiproducción. La red ofrece o no varios bienes club; hay o no economías de alcance.

8. Costos de transacción. Existen o no, y en caso de existir, de qué tipo.

Dimensiones de la teoría de equipos

1. Objetivo. Acción conjunta de la red.

2. Financiación. ¿Cómo se distribuyen los costos y, de ser posible, las ganancias de la red entre los miembros?

3. Asimetrías de información. Problemas de información: selección adversa, azar moral.

4. Presencia de terceros. ¿ Otros agentes (diferentes de los miembros) participan en el funcionamiento de la red?

El cuadro 3 sintetiza el análisis de las dimensiones comunes a ambas teorías. El cuadro 4 muestra los resultados del análisis considerando las dimensiones de la teoría de clubes, y el cuadro 5, considerando las dimensiones de la teoría de equipos.

\section{SUGERENCIAS PARA MEJORAR LA OPERACIÓN DE LAS REDES}

De acuerdo con la teoría de clubes, el mecanismo de exclusión permite internalizar la congestión y asignar los recursos de manera más eficiente. En general las redes descritas usan un mecanismo de exclusión, excepto los clusters. Cuando no existe un mecanismo de exclusión no se puede determinar el tamaño óptimo del club y es posible que la red alcance el nivel de producción más eficiente. Es recomendable que los clusters adopten un mecanismo de exclusión que tenga en cuenta la heterogeneidad de los participantes.

Por otra parte, según esta teoría, en las redes cuyos miembros tienden a ser homogéneos es eficiente compartir equitativamente los costos, como sucede en los consorcios de exportación y, en cierta medida, en las redes de materiales de construcción. La asociación en consorcios de exportación tiende a ser óptima a medida que aumenta el número de miembros; y esto no siempre ocurre en los casos descritos. Según la teoría de equipos, cuando las empresas son adversas al riesgo (como parece ser el caso de los consorcios de exportación), la distribución equitativa de las ganancias fomenta la eficiencia e incentiva el esfuerzo óptimo. 




Revista de Economía Institucional, vol. i7, n.o 32, Primer semestre/2oi5, PP. 3i7-348 


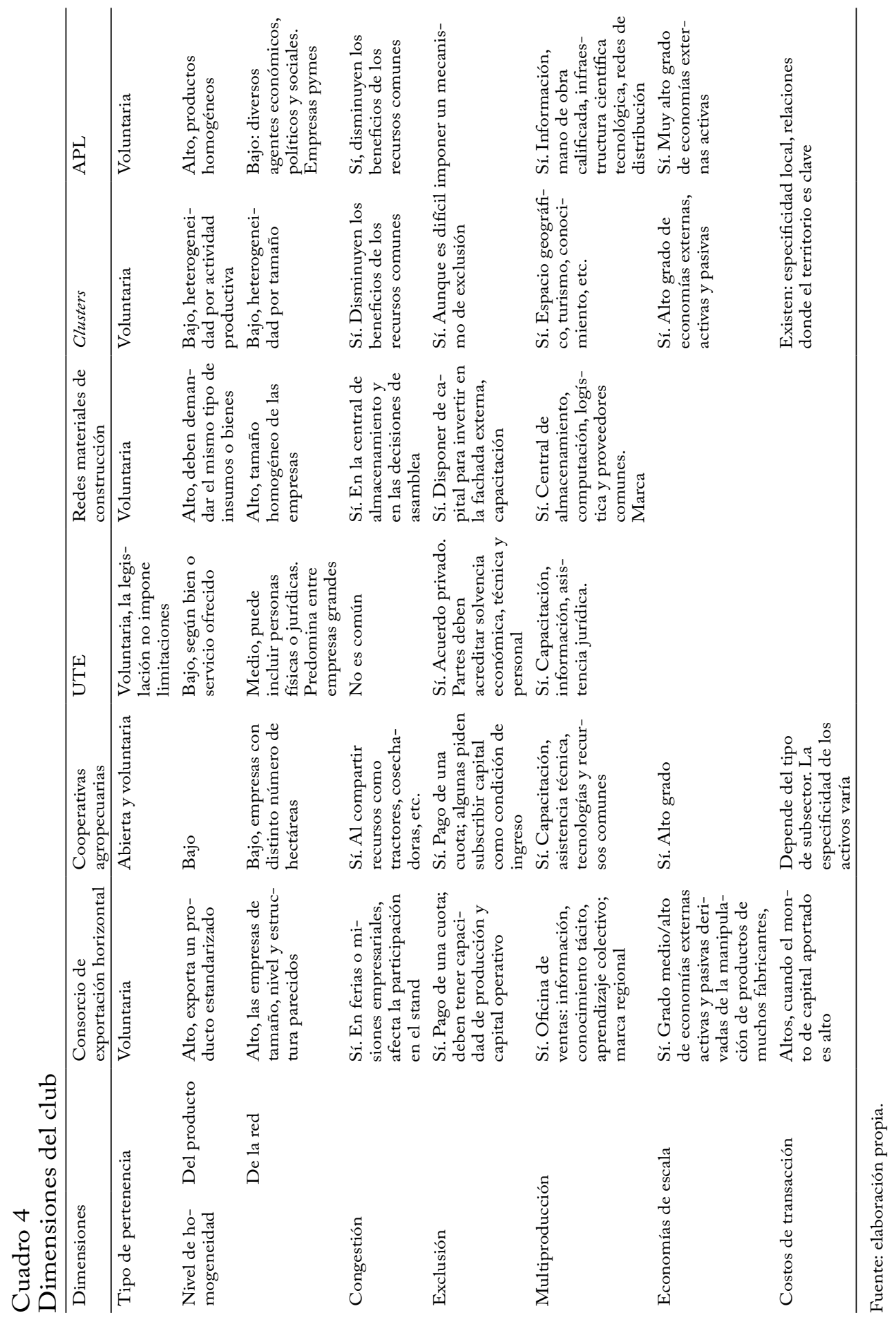

Revista de Economía Institucional, vol. i7, N.o 32, Primer semestre/2oi5, Pp. 3 I7-348 


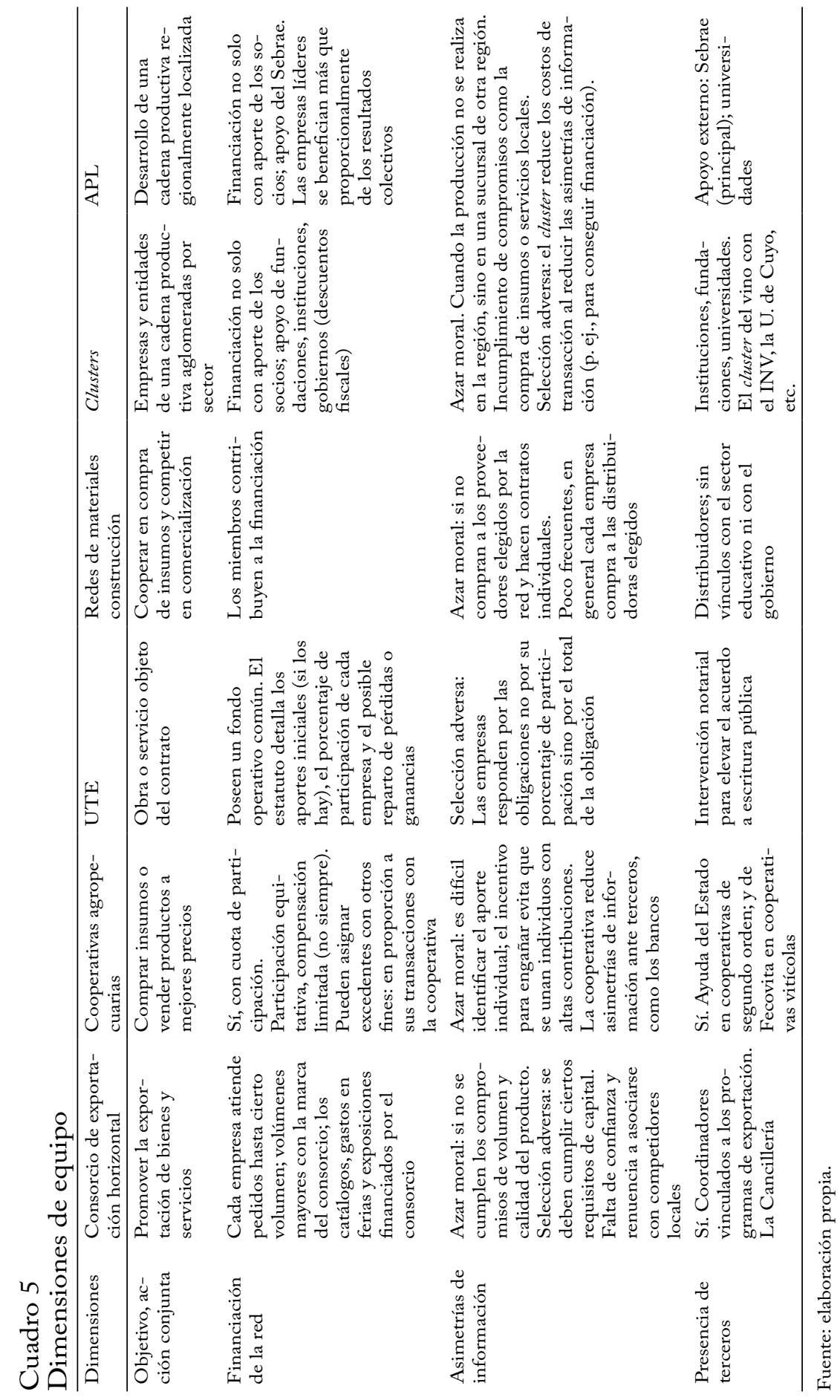

Revista de Economía Institucional, vol. i7, N. ${ }^{\circ} 32$, Primer semestre/2oi5, Pp. 3i 7-348 
La organización en cooperativas no es la forma ideal para resolver o atenuar los problemas de azar moral debidos a la dificultad de identificar el aporte de cada integrante. Desde el punto de vista del equipo, la cooperativa no evita el problema; y desde el punto de vista del club, una cuota de ingreso no igualitaria que tenga en cuenta la heterogeneidad de los aspirantes puede ofrecer una solución, además de un actor que oficie de principal para supervisar el desempeño.

Cuando se introducen diferencias entre los miembros, asimetrías de información y costos de transacción, la dimensión eficiente del club no está garantizada, se tiende a formar coaliciones, y algunos miembros pueden obtener rentas extraordinarias (Sandler y Tschirthart, 1997). En algunos casos, la teoría de equipos puede servir de marco para evaluar posibles soluciones a estos problemas.

Con respecto a los APL, la teoría de clubes permite incorporar en su tratamiento las dimensiones no económicas (cultural, entorno social, etc.) bajo la forma de multiproducción. Los clusters y los APL tienen problemas de asimetría de información y en general las empresas grandes sufren menos estas imperfecciones de mercado. Desde el punto de vista de la teoría de equipos, la presencia de terceros (servicios de apoyo, fundaciones) que financien bienes de uso común entre pymes de los APL produciría beneficios para todos.

\section{CONCLUSIONES}

Las redes de pymes se pueden analizar como clubes, equipos o ambos. La literatura sobre clubes y equipos se centra en los problemas de optimización, lo que dificulta su aplicación. Sin embargo, las teorías de clubes y de equipos son útiles para sistematizar algunas características observadas en las redes.

Los casos que aquí se estudian se analizaron primero como clubes y como equipos, pues las empresas que se unen en red pueden emprender un proyecto común (equipo) o usar recursos comunes (club). Ambas teorías ayudan a interpretar esos casos, y es difícil encontrar casos puros de club o de equipo. Los consorcios de exportación cuyo funcionamiento se asemeja al de un club, en cuanto comparten una oficina de ventas, también pueden emprender proyectos conjuntos, como exportar un producto con marca propia y garantía de calidad. Así la red de empresas cumple ambos fines y satisface las dimensiones de ambas teorías.

Conforme a la teoría de equipos, cada empresa participa en un proyecto conjunto y persigue su interés individual, lo cual la lleva a aprovechar en la mayor medida posible los resultados de la coope- 
ración. Así sucede, por ejemplo, cuando una red de farmacias une esfuerzos para crear un producto nuevo (un medicamento con marca de la red), pero sus integrantes aprovechan las ventajas de la organización colectiva haciendo contratos individuales con proveedores diferentes a los seleccionados por la red.

Por su parte, los bienes que suministran las redes a sus asociados suelen tener características de bienes club. En las redes descritas, los miembros comparten algunos recursos comunes, como la oficina de ventas en el caso de los consorcios de exportación o la central de almacenamiento en el caso de las redes de farmacias, que generan beneficios por la subaditividad de los costos. Los modelos de clubes ayudan a determinar el nivel de actividad óptimo (el volumen de exportación, p. ej.) y el tamaño del grupo (número de empresas). En la mayoría de los casos analizados, la medida del tamaño de la red es el número de miembros. En otros casos, como las UTE, el tamaño se refiere más a la tasa o frecuencia de uso, pues tienen pocos miembros generalmente y se forman para fines transitorios.

La alta posibilidad de exclusión de los bienes club mejora las perspectivas de financiación privada aunque esto depende de otros factores (como los niveles de inversión), lo que da margen para que la acción privada y la regulación pública interactúen y se garantice una provisión adecuada y eficiente del bien. Si las empresas (en particular las pymes) demandan bienes y servicios que tienen características de bienes club, la integración voluntaria en clubes es una alternativa eficiente para satisfacer esa demanda.

El aporte de este trabajo es el enfoque novedoso para analizar y caracterizar las redes y el tipo de alianzas entre pymes. Este enfoque no pretende sustituir a los que ya son bien conocidos y desarrollados para el análisis de las redes; es apenas complementario puesto que no incluye, por ejemplo, las dimensiones sociológicas de la cooperación, que son necesarias para una cabal interpretación de las redes. La investigación futura podría introducir la perspectiva dinámica en la teoría de clubes y analizar los costos de pertenecer a una red a medida que esta evoluciona. El trabajo se enriquecería con el estudio de la provisión voluntaria de bienes públicos o cuasi-públicos.

\section{REFERENCIAS}

1. Alderete, M. V. 2007. "Nuevas tecnologías de la información y de la comunicación: factores explicativos de la conducta exportadora en Argentina", Economía y Sociedad 12, 20, pp. 35-61. 
2. Andolfatto, D. y E. Nosal. "Optimal team contracts", The Canadian Journal of Economics 30, 2, 1997, pp. 385-396.

3. Bacic, M. y M. C. Souza. "Formación de redes horizontales de pequeñas empresas como estrategia de obtención de ventajas competitivas: análisis de una experiencia", Sciences de Gestion 66, 2008, pp. 215-232.

4. Boscherini, F. y L. Poma. "Más allá de los distritos industriales”, L. Poma, F. Boscherini y G. Yoguel, eds., Territorio, conocimiento y competitividad de las empresas: el rol de las instituciones en el espacio global, cap. 1, Madrid, Dávila editores, 2000.

5. Boschma, R. A. y J. G. Lambooy. "Knowledge, market structure, and economic coordination: Dynamics of industrial districts", Growth and Change 33, 2002, pp. 291-311.

6. Buchanan, J. "An economic theory of clubs", Economica 32, 125, 1965, pp. 1-14.

7. Che, Y. y S. Yoo. "Optimal incentives for teams", American Economic Review 91, 3, 2001, pp. 525-541.

8. Castellano, A; G. Ghezán y M. Mateos. "E1 rol del coordinador en los grupos de exportación de Pymes agroalimentarias”, IV Jornadas Interdisciplinarias de Estudios Agrarios y Agroindustriales, Buenos Aires, 2005.

9. Cendón, L.; G. Ghezán y M. Mateos. "Red de micro y pequeñas empresas alimentarias en la Provincia de Córdoba”, IV Jornadas Interdisciplinarias de Estudios Agrarios y Agroindustriales, Buenos Aires, 2005.

10. Cornes, R. y T. Sandler. The theory of externalities, public goods and club goods [1986], Nueva York, Cambridge University Press, 1996.

11. Costa C., M. T. "La cooperación entre empresas, nueva estrategia competitiva”, Economía Industrial 266, 1989, pp. 27-45.

12. Demange, G. y M. Wooders. Group formation in Economics. Networks, clubs and coalitions, Nueva York, Cambridge University Press, 2005.

13. Dini, M.; D. Mazzonis y R. Pérez. "Acciones colectivas: generación de confianza y cooperación para la competitividad”, FOMIN, Washington D.C., 2006.

14. Dini, M. “Competitividad, redes de empresas y cooperación empresarial”, ILPES Serie 76, Santiago de Chile, 2010.

15. Faulkner, D. "Strategic alliances: Cooperation for competition", D. Faulkner y G. Johnson, eds., The challenge of strategic management, Londres, Kogan Page, 1992, pp. 119-146.

16. Fundación BankBoston-Fundación Export.ar. "Informe de actividades. Programa para la formación de consorcios de exportación”, Buenos Aires, 2005.

17. Granovetter, M. "Economic action and social structure: The problem of embeddedness", American Journal of Sociology 91, 1985, pp. 481-510.

18. Hermosilla, A. y J. Solá. Cómo cooperar, Madrid, IMPI, 1990.

19. Holmstrom, B. "Moral hazard in teams", Bell Journal of Economics 13, 2, 1982, pp. 324-340.

20. Kantis, H., 2000. "Las redes de MIPyME y su rol en una estrategia de desarrollo empresarial: el caso de Rafaela”, III Foro Interamericano de la Microempresa, [www.iadb.org].

21. Koo, Y.-K. y Y. Seung-W. “Optimal incentives for teams”, American Economic Review 91, 3, 2001. 
22. Kosacoff, B. y A. López. “Cambios organizacionales y tecnológicos en las pequeñas y medianas empresas. Repensando el estilo de desarrollo argentino", Revista de la Escuela de Economía y Negocios 2, 4, 2000, pp. 42-49.

23. Kosacoff, B., ed. El desempeño industrial argentino. Más allá de la sustitución de importaciones, Buenos Aires, Cepal, 2000.

24. Laffont, J. J. y D. Martimort. The theory of incentives, Princeton University Press, 2002.

25. Mariti, P. "Los acuerdos de colaboración, entre empresas, en las economías modernas. Algunos apuntes para la reflexión”, Economía Industrial 266, 1989, pp. 61-68.

26. Mateos, M.; A. Castellano y M. Marino. "Diversidad productiva y estrategias empresarias en las pymes queseras: políticas a implementar para aumentar la competitividad", XV Reunión anual de la Asociación de Economía Agraria, Bahía Blanca, 2009.

27. McGuire, M. "Group segregation and optimal jurisdiction", Journal of Political Economy 82, 1, 1974, pp. 112-132.

28. McAfee, R. y J. McMillan. "Optimal contract for teams”, International Economic Review 32, 3, 1991, pp. 561-577.

29. Miles, R. E. y C. C. Snow. "Causes of failure in network organizations", California Management Review 34, 4, 1992, pp. 53-72.

30. Mirrlees, J. "An exploration in the theory of optimum income taxation", Review of Economic Studies 38, 1971, pp.175-208.

31. Montebugnoli, E. y R. Schiattarella. "Consideraciones en torno a los acuerdos entre empresas: particular referencia a las PME”, Economía Industrial 266, 1989, pp. 86-97.

32. Navarro, M. y A. Moreno. "El sector no lucrativo desde el enfoque de la elección pública: cuando la oferta no lucrativa de bienes públicos es el resultado de decisiones emanadas del proceso político", Revista de Economia Pública, Social y Cooperativa 37, 2001, pp. 117-140.

33. Olson, M. J. The logic of collective action: Public goods and the theory of groups, Cambridge, Mass., Harvard University Press, 1965.

34. Penrose, E. The theory of the growth of the firm, Nueva York, M. E. Sharpe, 1959.

35. Perroux, F. A economia do século XX, Lisboa, Herder, 1967.

36. Podolny, J. y K. Page. "Networks forms of organization", Annual Review of Sociology 24, 1998, pp. 57-76.

37. Rauch, J. y A. Casella. Networks and markets, Nueva York, Russell Sage Foundation, 2001.

38. Rasmussen, E. "Moral hazard in risk averse teams", RAND Journal of Economics 18, 3, 1987, pp. 428-435.

39. Sandler, T. y J. Tschirhart. "The economic theory of clubs: An evaluative survey”, Journal of Economic Literature 18, No. 4, 1980, pp. 1481-1521.

40. Sandler, T. y J. Tschirhart. "Club theory: Thirty years later", Public Choice 93, 1997, pp. 335-355.

41. Sandler, T.; F. P. Sterbenz y J. Tschirhart. "Uncertainty and clubs", Economica, N. S. 52, 208, 1985, pp. 467-477.

42. Schmitz, H. "Does local cooperation matter? Evidence from industrial clusters in South Asia y Latin America", Institute of Development Studies, University of Sussex, Brighton, 1999. 
43. Storper, M. y B. Harrison. "Flexibility, hierarchy and regional development: The changing structure of industrial production systems and their forms of governance in the 1990s", Research Policy 20, 5, 1991, pp. 407-422.

44. Tiebout, C. M. "A pure theory of local expenditures", Journal of Political Economy 64, 5, 1956, pp. 416-24.

45. Vander Veen, T. "Optimal contracts for teams: A note on the results of McAfee and McMillan”, International Economic Review 36, 4, 1995, pp. 1051-1056.

46. Vickrey, W. "Counter-speculation, auctions and competitive sealed tenders", Journal of Finance 16, 1961, pp. 8-37.

47. Yoguel, G. y F. Boscherini. "La capacidad innovativa y el fortalecimiento de la competitividad de las firmas: el caso de las Pymes exportadoras argentinas", Buenos Aires, Cepal, documento de trabajo 71, 1996.

48. Williamson, O. Las instituciones económicas del capitalismo, México D.F., Fondo de Cultura Económica, 1985. 